\title{
FITOSSOCIOLOGIA DE SUB-BOSQUE DE CERRADO EM TALHÃO DE Eucalyptus grandis W. Hill ex Maiden NO MUNICÍPIO DE BOM DESPACHO-MG ${ }^{1}$
}

\author{
Amilcar Walter Saporetti Jr. ${ }^{2}$, João Augusto Alves Meira Neto ${ }^{3}$ e Roosevelt Almado ${ }^{4}$
}

\begin{abstract}
RESUMO - O Cerrado, o segundo maior bioma brasileiro, apresenta uma crescente área sujeita a impactos variados, o que desperta a necessidade de conservá-lo e desenvolver métodos para seu uso sustentável. Os objetivos do presente trabalho foram realizar um levantamento florístico e fitossociológico de regeneração em um Cerrado no município de Bom Despacho - MG e embasar tomadas de decisões para conservação da biodiversidade. Para o estudo foram instaladas 15 parcelas de $200 \mathrm{~m}^{2}$ (10 x 20 m) em uma área originalmente de Cerrado, que atualmente encontra-se ocupada por um plantio de Eucalyptus grandis. A distribuição das parcelas foi feita sistematicamente. Foram amostradas todas as plantas lenhosas eretas com CAB - ao nível do solo - maior ou igual a $10 \mathrm{~cm}$. A composição florística apresentou 39 espécies, distribuídas em 24 famílias. As famílias com maior número de espécies foram Leguminosae Caesalpinioideae, com cinco espécies, Myrtaceae, com quatro, e Melastomataceae, com três, seguidas de Annonaceae, Leguminosae Papilionoideae, Nyctaginaceae, Rubiaceae e Vochysiaceae, com duas. A espécie que apresentou o maior VI foi Eucalyptus grandis (42,03\%), seguida das Miconia albicans (7,69\%), Brosimum gaudichaudii (6,9\%), Eugenia dysenterica (4,99\%), Qualea grandiflora (3,11\%) e Xylopia aromatica (3,01\%). Das 39 espécies lenhosas nativas encontradas no sub-bosque de Eucalyptus grandis, 27 são peculiares do Cerrado e as demais são acessórias, vindas de florestas da região. Esses dados mostram que o Cerrado está em regeneração.
\end{abstract}

Palavras-chave: Sub-bosque de Eucalyptus grandis, dispersão, Cerrado e savana.

\section{PHYTOSOCIOLOGY OF A CERRADO UNDERSTORY IN A STAND OF Eucalyptus grandis W. Hill ex Maiden IN BOM DESPACHO-MG}

\begin{abstract}
Cerrado, the second largest Brazilian bioma, is an area increasingly subject to various impacts, thus demanding conservation and development of sustainable use methods. The objective of this work was to conduct a floristic and phytosociological regeneration survey of a Cerrado in Bom Despacho-MG as a basis for biodiversity and conservation - oriented decision - making. Thus, 15 plots of $200 \mathrm{~m}^{2}(10 \times 20 \mathrm{~m})$ were established in a former Cerrado area currently occupied by Eucalyptus grandis stands. Plots distribution was systematically performed. All the standing woody plants with a ground level circumference of $10 \mathrm{~cm}$ or more were sampled. The floristic composition presented 39 species distributed within 24 families. The families with greater number of species were Leguminosae Caesalpinioideae with 5 species, Myrtaceae with 4, Melastomataceae with 3, followed by Annonaceae, Leguminosae Papilionoideae, Nyctaginaceae, Rubiaceae and Vochysiaceae, with 2 species. The species presenting the greatest VI was Eucalyptus grandis (42,03\%). The native species which presented greater VI were Miconia albicans (7,69\%), Brosimum gaudichaudii (6,9\%), Eugenia dysenterica (4,99\%), Qualea grandiflora $(3,11 \%)$ and Xylopia aromatica $(3,01 \%)$. Out of the 39 native woody species within the understory of Eucalyptus grandis, 27 are typical of Cerrado; the others are acessory species which came from forests in the region. These data show that this Cerrado region is being regenerated.
\end{abstract}

Key words: $\quad$ Eucalyptus grandis understory, dispersal, Cerrado vegetation, savannah.

1 Recebido para publicação em 7.8.2002.

Aceito para publicação em 4.11.2003.

2 Pós-graduação em Botânica, Universidade Federal de Viçosa - UFV, <saporetti@ vicosa.ufv.br>; ${ }^{3}$ Prof. do Departamento de Biologia Vegetal -UFV, <j.meira@ mail.ufv.br>. ${ }^{4} \mathrm{CAF}$ - Santa Bárbara Ltda. 
O Cerrado em Minas Gerais tem sido objeto de discussão de grupos temáticos que estudam a conservação da biodiversidade no Estado. Dentre as áreas que possuem vegetação de Cerrado a denominada Bom Despacho, que engloba os municípios de Bom Despacho, Martinho Campos e Dores do Indaiá, foi definida como Área de Alta Importância Biológica. Como resultado, foram estabelecidas recomendações para conservação de sua biodiversidade. Essas recomendações são, pela ordem, criação de unidades de conservação, investigação científica e recuperação de áreas degradadas (BIODIVERSIDADE ..., 1998). A região de Bom Despacho possui uma extensa área com povoamentos de Eucalyptus spp. para produção de madeira e carvão. Camargo (1998) salientou que em muitas plantações de Eucalyptus spp., em todo o Brasil, tem sido observada e considerada comum a formação de um denso sub-bosque de espécies nativas a partir de regeneração natural. $\mathrm{O}$ estabelecimento dessas espécies pode partir de diásporos advindos de vegetação autóctone vizinha aos plantios, do banco de sementes presentes no solo local ou da brotação de órgãos subterrâneos gemíferos, fato comum após a implantação de povoamentos florestais em áreas de Cerrado (Aubert \& Oliveira Filho, 1994).

Os objetivos deste trabalho foram realizar um levantamento florístico e fitossociológico de uma regeneração de Cerrado no município de Bom Despacho e embasar tomadas de decisões para conservação e manejo da biodiversidade de Cerrado em plantios de Eucalyptus abandonados.

A área de estudos encontra-se no Centro de Educação Ambiental - CEAM - da empresa CAF, em Santa Bárbara. Trata-se de um talhão de Eucalyptus grandis abandonado, mas que atualmente destina-se ao manejo e à conservação da biodiversidade.

Essa área localiza-se no município de Bom Despacho, nas coordenadas $19^{\circ} 41^{\prime} \mathrm{S}$ e $45^{\circ} 22^{\prime} \mathrm{W}$, cuja altitude é de $695 \mathrm{~m}$. Possui clima do tipo Cwa, pelo sistema de Köppen, e a precipitação média anual é de $1.400 \mathrm{~mm}$.

O solo é do tipo Latossolo Vermelho distrófico, e o trecho de vegetação estudado fica em um interflúvio pouco ondulado, local em que foi feito um plantio de Eucalyptus grandis em 1972 e o último corte foi realizado em 1990. O presente trabalho foi realizado durante o ano de 2000.

O levantamento florístico considerou as espécies lenhosas amostradas nas parcelas. A identificação taxonômica foi efetuada mediante consultas a herbários, consultas a especialistas e por meio de literatura especializada. $\mathrm{O}$ sistema de classificação utilizado foi o de Cronquist (1988).

Foi amostrado 0,3 ha de área total mediante a instalação de 15 parcelas de $200 \mathrm{~m}^{2}(10 \times 20 \mathrm{~m})$. A distribuição das parcelas foi feita de maneira sistemática ao longo de trilhas, distanciadas $20 \mathrm{~m}$ entre si.

Foram amostradas todas as plantas lenhosas eretas com circunferência do caule à altura da base $(\mathrm{CAB})$ - ao nível do solo - maior ou igual a $10 \mathrm{~cm}$.

Os parâmetros fitossociológicos calculados foram densidade, frequiência, dominâncias absoluta e relativa e, a partir dessas, o valor de importância (MuellerDombois \& Ellenberg, 1974). Foram calculados o índice de diversidade de Shannon (H') e a equabilidade (J') para a amostra (Pielou, 1975), como indicadores de heterogeneidade. Para calcular os referidos parâmetros foi utilizado o software Fitopac 1 (Shepherd, 1996).

Foram identificadas 39 espécies, pertencentes a 24 famílias (Quadro 1). As famílias com maior número de espécies foram Leguminosae Caesalpinioideae, com cinco espécies, Myrtaceae, com quatro, e Melastomataceae, com três, seguidas de Annonaceae, Leguminosae Papilionoideae, Nyctaginaceae, Rubiaceae e Vochysiaceae, com duas cada. (Quadro 1).

Segundo os parâmetros fitossociológicos, há dominância da família Myrtaceae na vegetação, seguida de Melastomataceae, Moraceae, Annonaceae, Vochysiaceae, Leguminosae Caesalpinioideae, Leguminosae Papilionoideae, Monimiaceae, Malpighiaceae e Ebenaceae.

Para as espécies, o índice de diversidade de Shannon (H') calculado foi de 2,636 e a equabilidade (J') foi de 0,715 , considerados baixos quando comparados aos de Cerrados bem conservados. Tal resultado se justifica pela presença de Eucalyptus grandis no local.

Comparativamente, a espécie que apresentou a maior densidade relativa (DR) e valor de importância (VI) foi Eucalyptus grandis (46,03\%). As espécies de Cerrado que apresentaram maior densidade relativa foram Miconia albicans $(12,15 \%)$ e Brosimum gaudichaudii $(11,09 \%)$. Ambas mostraram uma freqüência absoluta igual à do Eucalyptus grandis, 86,67\% (Quadro 2).

As espécies que apresentaram maior dominância relativa foram Eucalyptus grandis (86,37\%), Miconia albicans (2,74\%), Brosimum gaudichaudii e Xylopia aromatica $(1,44 \%)$ e Eugenia dysenterica $(1,24 \%)$ (Quadro 2). 
Quadro 1 - Lista de espécies lenhosas de Cerrado observadas em regeneração sob povoamento de $E$. grandis no município de Bom Despacho-MG

Table 1 - Woody species list of Cerrado being regenerated under E. grandis in Bom Despacho-MG

\begin{tabular}{|c|c|c|c|}
\hline $\mathrm{N}^{\mathrm{o}}$ & Família & Espécie & Nome Vulgar Local \\
\hline \multirow{4}{*}{1} & \multirow{4}{*}{ ANNONACEAE } & Annona coriacea Mart. & \\
\hline & & Annona dioica A.St. - Hil. & Araticum/marolo \\
\hline & & Duguetia furfuracea (A. St. - Hil.) Saff. & \\
\hline & & Xylopia aromatica (Lam.) Mart. & Pindaíba \\
\hline 2 & ARALIACEAE & Didymopanax macrocarpum Seem. & Mandioqueira \\
\hline 3 & ASTERACEAE & Piptocarpha rotundifolia (Less.) Baker & Macieira \\
\hline 4 & BOMBACACEAE & $\begin{array}{l}\text { Eriotheca pubescens (Mart. \& Zucc.) Schott \& } \\
\text { Endl. }\end{array}$ & Paineira-do-campo \\
\hline 5 & CHRYSOBALANACEAE & $\begin{array}{l}\text { Couepia grandiflora (Mart. \& Zucc.) Benth. ex } \\
\text { Hook. f. }\end{array}$ & \\
\hline 6 & CLUSIACEAE & Kielmeyera coriacea (Spreng.) Mart. & Pau-santo \\
\hline 7 & EBENACEAE & Diospyros hispida A.DC. & Caqui-do-cerrado \\
\hline 8 & FLACOURTIACEAE & Casearia decandra Jacq. & Pau-de-lagarto/erva-lagarto \\
\hline 9 & LAURACEAE & Ocotea pulchella Mart. & Canela \\
\hline \multirow{5}{*}{10} & \multirow{5}{*}{$\begin{array}{l}\text { LEGUMINOSAE } \\
\text { CAESALPINIACEAE }\end{array}$} & Acosmium dasycarpum (Vogel) Yakovlev & Chapada \\
\hline & & Acosmium subelegans (Mohlenbr.) Yakovlev & \\
\hline & & Bauhinia holophylla Steud. & Pata-de-vaca/unha-de-bezerro \\
\hline & & Sclerolobium paniculatum Vogel & Angá \\
\hline & & Senna rugosa (G. Don) Irwin \& Barneby & Canafístula \\
\hline \multirow{2}{*}{11} & \multirow{2}{*}{$\begin{array}{l}\text { LEGUMINOSAE } \\
\text { PAPILIONOIDEAE }\end{array}$} & Dalbergia miscolobium Benth. & Caviúna \\
\hline & & Machaerium opacum Vogel & Jacarandá-roxo \\
\hline 12 & MALPIGHIACEAE & Byrsonima intermedia A. Juss. & Muricizinho \\
\hline \multirow{3}{*}{13} & \multirow{3}{*}{ MELASTOMATACEAE } & Miconia albicans (Sw.) Triana & $\mathrm{Cu}$-de-pinto \\
\hline & & Miconia langsdorffii Cogn. & Quaresminha/quaresmeira \\
\hline & & Miconia sellowiana Naudin & Quaresminha/quaresmeira \\
\hline 14 & MELIACEAE & Cabralea canjerana (Vell.) Mart. & Canjerana \\
\hline 15 & MONIMIACEAE & Siparuna guianensis Aubl. & Catinga-d'anta \\
\hline 16 & MORACEAE & Brosimum gaudichaudii Trécul & Mamica-de-cadela \\
\hline \multirow{4}{*}{17} & \multirow{4}{*}{ MYRTACEAE } & Eugenia aurata O.Berg & \\
\hline & & Eugenia dysenterica DC. & Cagaita \\
\hline & & Myrcia lingua (O. Berg) Mattos \& Legrand & Goiabinha \\
\hline & & Myrcia rostrata $\mathrm{DC}$. & Folha-miúda \\
\hline 18 & MYRSINACEAE & Rapanea ferruginea (Ruíz \& Pav.) Mez & Pororoca \\
\hline 19 & MYRISTICACEAE & Virola sebifera Aubl. & Café-do-mato \\
\hline \multirow{2}{*}{20} & \multirow{2}{*}{ NYCTAGINACEAE } & Guapira noxia (Netto) Lundell & Maria-mole \\
\hline & & Neea theifera Oerst. & \\
\hline 21 & OCHNACEAE & Ouratea spectabilis (Mart. ex Engl.) Engl. & Gordinha \\
\hline \multirow{2}{*}{22} & \multirow{2}{*}{ RUBIACEAE } & Palicourea rigida Kunth & Bate-caixa \\
\hline & & Rudgea viburnoides (Cham.) Benth. & Erva-de-bugre \\
\hline 23 & SYMPLOCACEAE & Symplocos cf. pubescens Klotzsch ex Benth. & \\
\hline \multirow{2}{*}{24} & \multirow{2}{*}{ VOCHYSIACEAE } & Qualea grandiflora Mart. & Pau-terra \\
\hline & & Qualea multiflora Mart. & Pau-terrinha \\
\hline
\end{tabular}


Quadro 2 - Parâmetros fitossociológicos do sub-bosque - CEAM - Bom Despacho-MG Table 2 - Understory phytosociological parameters - CEAM - Bom Despacho-MG

\begin{tabular}{|c|c|c|c|c|c|c|c|}
\hline Espécie & DA & DR & FA & FR & DoA & DoR & VI \\
\hline Eucalyptus grandis & 493,3 & 31,56 & 86,67 & 8,18 & 22,9977 & 86,37 & 126,11 \\
\hline Miconia albicans & 190,0 & 12,15 & 86,67 & 8,18 & 0,7288 & 2,74 & 23,07 \\
\hline Brosimum gaudichaudii & 173,3 & 11,09 & 86,67 & 8,18 & 0,3828 & 1,44 & 20,70 \\
\hline Eugenia dysenterica & 106,7 & 6,82 & 73,33 & 6,92 & 0,3295 & 1,24 & 14,98 \\
\hline Qualea grandiflora & 66,7 & 4,26 & 46,67 & 4,40 & 0,1763 & 0,66 & 9,33 \\
\hline Xylopia aromatica & 50,0 & 3,20 & 46,67 & 4,40 & 0,3829 & 1,44 & 9,04 \\
\hline Miconia langsdorffii & 70,0 & 4,48 & 26,67 & 2,52 & 0,1736 & 0,65 & 7,65 \\
\hline Annona coriacea & 26,7 & 1,71 & 46,67 & 4,40 & 0,0654 & 0,25 & 6,35 \\
\hline Myrcia lingua & 30,0 & 1,92 & 40,00 & 3,77 & 0,0654 & 0,25 & 5,94 \\
\hline Siparuna guianensis & 26,7 & 1,71 & 40,00 & 3,77 & 0,0645 & 0,24 & 5,72 \\
\hline Byrsonima intermedia & 30,0 & 1,92 & 33,33 & 3,14 & 0,1498 & 0,56 & 5,63 \\
\hline Dalbergia miscolobium & 26,7 & 1,71 & 33,33 & 3,14 & 0,0657 & 0,25 & 5,10 \\
\hline Diospyros hispida & 20,0 & 1,28 & 33,33 & 3,14 & 0,0380 & 0,14 & 4,57 \\
\hline Virola sebifera & 23,3 & 1,49 & 26,67 & 2,52 & 0,1209 & 0,45 & 4,46 \\
\hline Acosmium subelegans & 16,7 & 1,07 & 33,33 & 3,14 & 0,0311 & 0,12 & 4,33 \\
\hline Miconia sellowiana & 16,7 & 1,07 & 26,67 & 2,52 & 0,0802 & 0,30 & 3,88 \\
\hline Acosmium dasycarpum & 16,7 & 1,07 & 26,67 & 2,52 & 0,0359 & 0,13 & 3,72 \\
\hline Piptocarpha rotundifolia & 13,3 & 0,85 & 26,67 & 2,52 & 0,0529 & 0,20 & 3,57 \\
\hline Myrcia rostrata & 23,3 & 1,49 & 20,00 & 1,89 & 0,0480 & 0,18 & 3,56 \\
\hline Qualea multiflora & 16,7 & 1,07 & 20,00 & 1,89 & 0,0662 & 0,25 & 3,20 \\
\hline Symplocos cf. pubescens & 10,0 & 0,64 & 20,00 & 1,89 & 0,0551 & 0,21 & 2,73 \\
\hline Guapira noxia & 10,0 & 0,64 & 20,00 & 1,89 & 0,0215 & 0,08 & 2,61 \\
\hline Didymopanax macrocarpum & 10,0 & 0,64 & 13,33 & 1,26 & 0,1129 & 0,42 & 2,32 \\
\hline Palicourea rigida & 23,3 & 1,49 & 6,67 & 0,63 & 0,0437 & 0,16 & 2,29 \\
\hline Machaerium opacum & 10,0 & 0,64 & 13,33 & 1,26 & 0,0157 & 0,06 & 1,96 \\
\hline Eugenia aurata & 6,7 & 0,43 & 13,33 & 1,26 & 0,0259 & 0,10 & 1,78 \\
\hline Senna rugosa & 6,7 & 0,43 & 13,33 & 1,26 & 0,0077 & 0,03 & 1,71 \\
\hline Rudgea viburnoides & 6,7 & 0,43 & 13,33 & 1,26 & 0,0071 & 0,03 & 1,71 \\
\hline Ouratea spectabilis & 6,7 & 0,43 & 13,33 & 1,26 & 0,0065 & 0,02 & 1,71 \\
\hline Sclerolobium paniculatum & 3,3 & 0,21 & 6,67 & 0,63 & 0,1961 & 0,74 & 1,58 \\
\hline Eriotheca pubescens & 3,3 & 0,21 & 6,67 & 0,63 & 0,0344 & 0,13 & 0,97 \\
\hline Couepia grandiflora & 3,3 & 0,21 & 6,67 & 0,63 & 0,0106 & 0,04 & 0,88 \\
\hline Rapanea ferruginea & 3,3 & 0,21 & 6,67 & 0,63 & 0,0068 & 0,03 & 0,87 \\
\hline Bauhinia holophylla & 3,3 & 0,21 & 6,67 & 0,63 & 0,0052 & 0,02 & 0,86 \\
\hline Casearia decandra & 3,3 & 0,21 & 6,67 & 0,63 & 0,0045 & 0,02 & 0,86 \\
\hline Cabralea canjerana & 3,3 & 0,21 & 6,67 & 0,63 & 0,0038 & 0,01 & 0,86 \\
\hline Kielmeyera coriacea & 3,3 & 0,21 & 6,67 & 0,63 & 0,0038 & 0,01 & 0,86 \\
\hline Annona dioica & 3,3 & 0,21 & 6,67 & 0,63 & 0,0038 & 0,01 & 0,86 \\
\hline Ocotea pulchella & 3,3 & 0,21 & 6,67 & 0,63 & 0,0026 & 0,01 & 0,85 \\
\hline Duguetia fufuracea & 3,3 & 0,21 & 6,67 & 0,63 & 0,0026 & 0,01 & 0,85 \\
\hline
\end{tabular}

DA-densidade absoluta, DR-densidade relativa, FA-frequiência absoluta, FR-frequêencia relativa, DoA-dominância absoluta, DoR-dominância relativa e VI-valor de importância.

$D A$ - Absolute density; DR - Relative density; FA - Absolute frequency; FR - Relative frequency; DoA - Absolute dominance; DoR - Relative dominance; VI-Importance value. 
As espécies que apresentaram o maior VI foram Miconia albicans (7,67\%), Brosimum gaudichaudii (6,9\%), Eugenia dysenterica (4,99\%), Qualea grandiflora $(3,11 \%)$ e Xylopia aromatica $(3,01 \%)$ (Quadro 2).

Miconia albicans, Brosimum gaudichaudii, Eugenia dysenterica, Qualea grandiflora, Xylopia aromatica, Miconia langsdorffii, Annona coriacea, Myrcia lingua, Byrsonima intermedia, Dalbergia miscolobium e Diospyros hispida são espécies que demonstraram capacidade de estabelecimento em sub-bosque de Eucalyptus grandis.

Das 39 espécies lenhosas que se estabeleceram no sub-bosque de Eucalyptus grandis, 27 são peculiares do Cerrado; as demais são acessórias, vindas de Florestas Estacionais Semideciduais da região, fenômeno comum na flora do Cerrado.

Em termos de riqueza florística, destacam-se Leguminosae Caesalpinioideae com cinco espécies, Myrtaceae com quatro e Melastomataceae com três. Sete famílias contribuíram com apenas um indivíduo.

A regeneração de Cerrado em áreas selecionadas de plantio de Eucalyptus para manejo e conservação da biodiversidade no CEAM, quando comparada com um Cerrado sensu stricto bem conservado em Abaeté (Meira Neto et al., 2003), mostra que Eucalyptus grandis ocupou grande parte da capacidade de suporte do local e apresentou $22 \mathrm{~m}^{2} /$ ha de área basal. Para comparação, no Cerrado de Abaeté Caryocar brasiliensis foi a população de maior área basal, com 3,69 m²/ha (Meira Neto et al., 2003). Ao comparar a estrutura do Cerrado de Abaeté com o sub-bosque no talhão do CEAM em Bom Despacho, constata-se que as populações mais sensíveis à ocupação da capacidade de suporte por Eucalyptus grandis foram Caryocar brasiliensis, Xylopia aromatica, Eugenia dysenterica, Myrcia lingua, Brosimum gaudichaudii, Erythroxylum daphnites e Qualea grandiflora, todas espécies peculiares de Cerrado. Todas estas são polinizadas por insetos. Com exceção de Qualea grandiflora, todas são dispersas por vertebrados, notadamente pássaros e mamíferos (Gottsberger \& Silberbauer-Gottsberger, 1983). Estas populações tiveram áreas basais menores no sub-bosque de Eucalyptus grandis. Confrontando esses resultados com os de Cerrado sensu stricto em Abaeté, percebe-se que houve diminuição de 0,6 a 3,6 $\mathrm{m}^{2}$ de área basal por hectare em cada uma dessas populações, retratando a menor capacidade de suporte para a fauna nativa no talhão do que em um Cerrado conservado. A única espécie nativa a se beneficiar dessa situação, Miconia albicans, é capaz de produzir sementes por apomixia, sem polinização (Goldenberg \& Shepherd, 1998), e embora seja dispersa por aves teve uma área basal de $0,55 \mathrm{~m}^{2} / \mathrm{ha}$ no Cerrado de Abaeté e $0,73 \mathrm{~m}^{2} /$ ha no sub-bosque de Eucalyptus grandis, representando um aumento de apenas $0,18 \mathrm{~m}^{2} / \mathrm{ha}$. $\mathrm{O}$ conjunto indica que a área basal de $22 \mathrm{~m}^{2} /$ ha que Eucalyptus grandis tem na área do CEAM representa a utilização da maior parte dos nutrientes antes disponíveis para a flora nativa.

Seriam ações recomendáveis para a completa recuperação da vegetação de Cerrado no sub-bosque de Eucalyptus em Bom Despacho o anelamento das árvores de Eucalyptus grandis e a manutenção dessas árvores mortas em pé, pois haveria a reposição para o Cerrado dos nutrientes pela decomposição dessa matéria orgânica e, principalmente, as árvores mortas em pé seriam perfeitos poleiros para aves, o que permitiria que estas trouxessem diásporos das populações de espécies dispersas por pássaros. É o caso das populações de Xylopia aromatica, Eugenia dysenterica, Myrcia lingua, Brosimum gaudichaudii e Erythroxylum daphnites (Gottsberger \& Silberbauer-Gottsberger, 1983). Essa ação permitiria uma efetiva recuperação da composição florística e da estrutura do Cerrado local, aproximandoas das de outros Cerrados bem conservados da região. A finalização da recuperação se daria com o plantio de mudas de Caryocar brasiliensis (Pequi) e de Qualea grandiflora; a primeira por não ter sido amostrada no CEAM e por não ser dispersa por aves, mas por outros vertebrados, e a segunda por ser dispersa pelo vento.

\section{REFERÊNCIAS BIBLIOGRÁFICAS}

AUBERT, E.; OLIVEIRA FILHO, A. T. Análise multivariada da estrutura fitossociológica do sub-bosque de Plantios Experimentais de Eucalyptus spp. e Pinus spp. em Lavras-MG. Revista Árvore, n. 18, n. 3, p. 194-214, 1994.

BIODIVERSIDADE em Minas Gerais: um atlas para sua conservação. Belo Horizonte: Fundação Biodiversitas, 1998. $94 \mathrm{p}$.

CAMARGO, S. L. Composição e estrutura fitossociológica da vegetação natural sob plantio de Eucalyptus grandis W. Hill ex Maiden no município de Dionísio-MG. 1998. $68 \mathrm{f}$. Dissertação (Mestrado em Ecologia - Conservação e Manejo da Vida Silvestre) - Universidade Federal de Minas Gerais, Belo Horizonte, 1998.

R. Árvore, Viçosa-MG, v.27, n.6, p.905-910, 2003 
CRONQUIST, A. The evolution and classification of flowering plants. New York : The New York Botanical Garden. 1988. 555 p.

GOLDENBERG, R.; SHEPHERD, G. J. Studies on the reproductive biology of Melastomataceae in "Cerrado" vegetation. Plant Systematics and Evolution, n. 211, p. 1329, 1998 .

GOTTSBERGER, G.; SILBERBAUER-GOTTSBERGER, I. Dispersal and distribution in the Cerrado Vegetation of Brazil. Sonderbd. Naturwiss. Ver. Hamburg, n. 7, p. 315352, 1983.
MEIRA NETO, J. A. A.; SAPORETTI JR. A. W.;

ALMADO, R. Fitossociologia de Cerrado sensu stricto no município de Abaeté, MG. Revista Árvore. No prelo, 2003.

MUELLER-DOMBOIS, D.; ELLENBERG, H. Aims and methods of vegetation ecology. New York: John Wiley \& Sons. 1974. $547 \mathrm{p}$

PIELOU, E. C. Ecological diversity. New York: John Wiley \& Sons. 1975. 165 p.

SHEPHERD, G. J. Fitopac 1. Manual do usuário Campinas: Universidade de Campinas, 1996. 80 p. 